RESEARCH ARTICLE

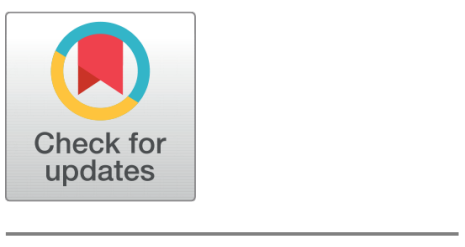

OPEN ACCESS

Received: 18.08.2021

Accepted: 20.10 .2021

Published: 22.11 .2021

Citation: Panchaxri , Jagadale BN, Priya BS, Nargund MN (2021) Image Denoising using Adaptive NL Means Filtering with Method Noise Thresholding. Indian Journal of Science and Technology 14(39): 2961-2970. https://doi.org/ 10.17485/IJST/v14i39.1532

* Corresponding author.

panchaksharih21@gmail.com

Funding: None

Competing Interests: None

Copyright: (c) 2021 Panchaxri et al. This is an open access article distributed under the terms of the Creative Commons Attribution License, which permits unrestricted use, distribution, and reproduction in any medium, provided the original author and source are credited.

Published By Indian Society for Education and Environment (iSee)

ISSN

Print: 0974-6846

Electronic: 0974-5645

\section{Image Denoising using Adaptive NL Means Filtering with Method Noise Thresholding}

\author{
Panchaxri $^{1 *}$, Basavaraj N Jagadale ${ }^{2}$, B S Priya ${ }^{3}$, Mukund N Nargund ${ }^{4}$ \\ 1 Department of Electronics, SSA Government First Grade College, Ballari, 583 101, India \\ 2 Department of Electronics, Kuvempu University, Shimoga, 577451, India \\ 3 Department of PG Studies and Research in Electronics, Kuvempu University, Shimoga, \\ 577451 , India \\ 4 Department of Physics and Electronics, Christ (Deemed to be University) University, \\ Bengaluru, 560029, India
}

\section{Abstract}

Background/Objectives: Image denoising is an important step in image processing applications. Usually noise is added to the original image during transmission, acquisition and storage process and is considered as noisy image. For precise analysis and extraction of image features, the noisy image is denoised without losing the original image details. This study aims to introduce a novel denoising method to obtain denoised image(s) such that it has fewer artifacts and is more efficient at higher noise levels. Method: The proposed novel denoising method introduces Adaptive Non Local Means (ANL) along with Method Noise Thresholding (MNT) technique to improve the image quality of the denoised image. Method Noise (MN) image obtained by taking the difference of image details between noisy image and pre-filtered mage. Recovered value from the MN through thresholding includes some of the important components of the original image. These values computed added to pre-filtered image to recover image features of the original image. Findings: The standard image, denoised with noise standard $(\sigma=10)$ using bior6.8 wavelet when filtered using existing Gaussian Bilateral Filter along with Method- Noise Thresholding filtering technique and Wiener Filter along with Residual Thresholding show improvement in quality of the denoised image in terms of PSNR and ISSN values as compared to the proposed filtering technique. The proposed filter technique results in higher PSNR and ISSN values (PSNR $=33.80$ and SSIN $=0.9994$ ). Novelty: It is known that ANLM results in improved denoised parameters compared with NLM filter; however, when MNT is blended with ANLM shows further improvement in quality of denoised image. Hence, in the proposed method, MNT is incorporated along with ANLM for improvement in denoising process. Image Quality Index (IQI) of the different standard images using ANLMT filtering technique is also studied.

Keywords: Adaptive NonLocal Means filter; Gaussian Filter; Method Noise; Wavelet Thresholding; WienerFilter; and Adaptive Non Local Means Filter with Method Noise Thresholding 


\section{Introduction}

Image processing is in practice in most of the medical, industrial, and military applications. In this process, for accurate analysis of the image(s) by human interpretation or autonomous machine perception, denoising of the image is mandatory. Denoising process helps to obtain original image from the corrupted image. Continuous efforts made by the researchers in this field to improve coding technique or introduce new filtering methods to get better-denoised images in terms of retaining or recovering original details of the image. Various denoising technique are in use. Improved Non Local Means (NLM) and other denoising techniques proposed in recent years ${ }^{(1-4)}$.

Quality of denoised image improves by wavelet-based denoising technique. Wavelet-based image denoising techniques estimate the threshold value by considering either subband or universal thresholding to denoise the image. Visushrink, SURE shrink, Bayes shrink, and Neighsure shrink are some of the thresholding methods to estimate the threshold value. B. K. Shreyamsha Kumar proposed ${ }^{(5)}$ Gaussian Filter-based method noise thresholding [GBFMT] combines Gaussian filter and wavelet thresholding technique using Bayes thresholding. This method suitable at lower noise levels but produces some artifacts at higher noise levels. Priya B.S ${ }^{(6)}$ introduce the Weiner filter-based residual noise thresholding [WFRT] method uses Wiener filter and Neighsure shrink wavelet thresholding. WFRT filter has improvement in denoised image quality over GBFMT filter but fails to denoise efficiently at higher noise levels. In this work, a novel denoising technique in which Neighsure shrink wavelet thresholding is combined with adaptive non local means (ANLM) filter.

The paper provides brief introduction of image filters and use of these filters along with different thresholding methods in section 1. Different thresholding techniques discussed in details in section 2. ANLM filter adopted in the present work briefly discussed in section 3. Section 4 deals with methodology followed in this paper through the block diagram along with description of each block. Results and discussions described in the section 5. Section 6 concludes the proposed method.

\section{Thresholding Techniques}

\subsection{Visushrink}

The universal threshold defined by VisuShrinkis given in (1).

$$
T_{u}=\sigma_{n} \sqrt{2 \log (L)}
$$

Where $\sigma_{n}$ - noise standard deviation

and $\mathrm{L}$ - total number of pixels in an image.

However, this technique yields less preserved details since the threshold value is high for large values of $\mathrm{L}$, as it kills signal coefficients and noise.

\subsection{Sureshrink}

In the subband adaptive technique proposed by Donoho and Johnstone as Steins Unbiased Risk Estimate (SURE), it leads to select threshold TSURE, which adapts to the data by minimizing the estimation of the mean square error (MSE). Threshold parameter TSURE calculated using (2).

$$
\begin{gathered}
T_{S U R E}=\operatorname{argmin}_{T_{h}}\left(\operatorname{SURE}\left(T_{h} ; W\right)\right) \\
\left(\operatorname{SURE}\left(T_{h} ; W\right)=\sigma_{n}^{2}-\frac{1}{\mathrm{~L}} \times\left(2 \sigma_{\mathrm{n}}^{2}, \#\left\{\mathrm{i}:\left|\mathrm{W}_{\mathrm{i}}\right| \leq \mathrm{T}_{\mathrm{h}}-\sum_{i=1}^{L} \operatorname{mean}\left(\left|W_{i}\right|, T_{h}\right)^{2}\right\}\right)\right)
\end{gathered}
$$

Where, $\sigma_{n}^{2}$ - noise variance,

$\mathrm{L}$ - total number of wavelet coefficients of a subband under consideration,

$W_{i}$-wavelet coefficient of a subband,

$$
T_{h} \in\left|0, T_{u}\right|
$$

$T_{u}$-universal threshold 


\subsection{Bayeshrink}

For images corrupted by Gaussian noise, the BayesShrink method is more effective; it uses an adaptive data-driven threshold technique. In this method, soft thresholding and threshold of a sub band are determined by modeling the wavelet coefficients. Random variables are obtained within each subband of an image using Generalized Gaussian Distribution (GGD).

Bayes threshold $\left(\mathrm{T}_{B}\right)$ of a subband is calculated by (4).

$$
T_{B}=\frac{\sigma_{n}^{2}}{\sigma_{F}}
$$

Where $\sigma_{n}$ - noise variance

Here $\sigma_{n}$ estimated using a robust median estimator as given in (5).

$$
\sigma_{n}=\frac{\operatorname{median}|\{w i, j\} \in H H|}{0.6745}
$$

Where $\sigma_{F}$ - estimated signal standard deviation in wavelet domain

Here $\sigma_{F}$ is estimated using (6).

$$
\sigma_{F}=\sqrt{\max \left(\left(\sigma_{w}^{2}-\sigma_{n}^{2}\right), 0\right)}
$$

Where $\sigma_{w}^{2}$ - variance of w.

Where, $\mathrm{w}$ is modeled as zero mean, $\sigma_{w}^{2}$ is calculated as shown in (7).

$$
\sigma_{w}^{2}=\frac{1}{n^{2}} \sum_{j=1}^{n} w_{i, j}^{2}
$$

when $\sigma_{n}^{2}>\sigma_{w}^{2}, \sigma_{F}$ will become zero and $\mathrm{T}_{B}$ becomes $\infty$. For this case, the value of $\mathrm{T}_{B}$ is estimated using (8).

$$
T_{B}=\max \left(\left|w_{i, j}\right|\right)
$$

\subsection{Neighshrink}

This thresholding technique incorporates neighboring wavelet coefficients to estimate the threshold value. The neighborhood window size should be odd. A 3x3 window is depicted in Figure 1 below.

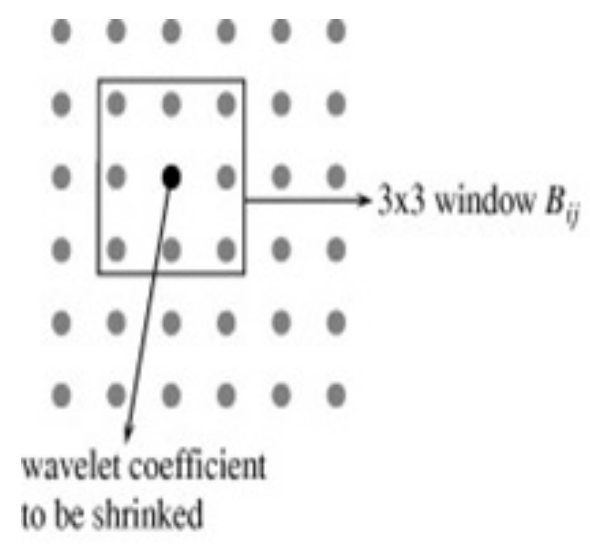

Fig 1. 3 x 3 Neighbor Window

The shrink function for a particular odd size window is given in (9).

$$
\beta_{i, j}=\left(1-\frac{T_{u}^{2}}{S_{i, j}^{2}}\right)_{+}
$$


$\mathrm{b}_{i, j}$ refers to centered wavelet coefficient.

Where, $T_{u}$ - universal threshold value

$\left(S_{i, j}\right)$ the sum of all the wavelet coefficients within the neighborhood window.

Where,

$$
S_{i, j}^{2}=\sum_{m=i-1}^{i+1} \sum_{n=j-1}^{j+1} w_{m, n}^{2}
$$

The + sign indicates to consider only positive values during estimation and becomes zero for negative values. The estimated center wavelet coefficient obtained through a noisy wavelet coefficient using (11).

$$
\widehat{w}_{i, j}=\beta_{i, j} w_{i, j}
$$

\subsection{Neighshrinksure (NSS)}

NeighShrinkSURE is an improved version of NeighShrink in the image denoising technique proposed by Dengwen and Wengang. Stein's Unbiased Risk Estimate (SURE) method used to determine each subband's optimal threshold and neighboring window size (12).

$$
\left(T^{S}, k^{S}\right)=\operatorname{argmin}_{T, k} S U R E\left(w_{S}, T, k\right)
$$

Where, $\mathrm{T}$ - threshold,

$\mathrm{k}$ - window size and

$\mathrm{s}$ - denotes the subband.

\section{Adaptive NL Means Filter}

Rajiv Verma et al. ${ }^{(7)}$ proposed sub window size in the Adaptive NL filter for denoising technique based on the average gray level difference(GLD) for particular window size. The larger the GLD, the lesser is the size of the search window due to significant variation in gray levels of non smooth regions. Images with smooth regions or areas with less gray-level variations result in lesser GLD and results in increase in size of the search window. This technique is helpful to improve the denoising quality of an image since it avoids the gray level of unrelated pixels while averaging in a fixed search window size. Here both denoising time and quality of an image are improved in this technique.

The mean gray level of a pixel value in the neighborhood window $\left(\mathrm{W}_{k}\right)$ of odd number $\mathrm{n} \mathrm{x} \mathrm{n}$ matrix, centered on pixel $\mathrm{i}$ is given as (13).

$$
\tilde{X}_{i}=\frac{1}{n x n} \sum_{k=1}^{n} \sum_{l=1}^{n} \hat{X}(k, l)
$$

Where, (k, l) - pixel coordinate in neighborhood $\Omega_{k}$.

The absolute gray level difference $\triangle \widetilde{X}_{i}$ between $\widetilde{X}_{i}$ and $\widetilde{X}_{i}$ for pixel 'i' is given by

$$
\left|\Delta \tilde{X}_{i}=\hat{X}_{i}-\tilde{X}_{i}\right|
$$

Gray level difference (GLD) of each pixel of an image calculated using (13) and (14).

The Mean $(\mu)$ of the GLD is calculated using the formula

$$
\mu=\frac{1}{M x N} \sum_{i \in \Delta \hat{X}_{i}} \Delta \hat{X}_{i}
$$

and standard deviation (s) of the GLD is calculated using the formula

$$
\sigma=\left[\frac{1}{M x N} \sum_{i \varepsilon \Delta \hat{X}_{i}}\left(\Delta \hat{X}_{i}-\mu\right)^{2}\right]^{\frac{1}{2}}
$$


Then threshold $\mathrm{T}_{1}$ and $\mathrm{T}_{2}$ are defined as

$$
\begin{gathered}
T_{1}=\mu \\
T_{2}=\mu+\alpha \sigma
\end{gathered}
$$

where $\alpha$ - control parameter.

Then an optimal search window can be made,

$$
S_{i}^{o p t}=\left\{\begin{array}{c}
\text { large, if } \Delta \widehat{X}_{l}<T_{1} \\
\text { medium, if } T_{1} \leq \Delta \widehat{X}_{l} \geq T_{2} \\
\text { small } \quad \text { if }, \Delta \widehat{X}_{l}>T_{2}
\end{array}\right.
$$

Denoised image obtained by adaptive search using the formula (20).

$$
\hat{X}_{\text {final }}(i)=\frac{\sum_{j \in S_{i}^{\mathrm{opt}} w(i, j) y(j)}}{\sum_{j \in S_{i}^{\mathrm{opt}} w(i, j)}}
$$

\section{Proposed Method}

Figure 2 depicts the complete block diagram of the proposed method. Primarily, the noisy image is filtered using an adaptive NL means filter to obtain denoised image $\mathrm{I}_{F}$. The filtered image and noisy images combined to generate method noise by subtracting filtered image $\mathrm{I}_{F}$ from noisy image. The residue after subtraction is called method noise. Residue value that comprises image details along with noise let to estimate the true wavelet coefficients. Wavelet decomposition was carried out to get all approximation and detail coefficients. Wavelet thresholding is employed to eliminate noise components in sub-bands. NeighshrinkSure technique gives better Mean Square Error (MSE). Finally, denoised image reconstructed by combining prefiltered image with the image details from Inverse Discrete Wavelet Transform (IDWT).

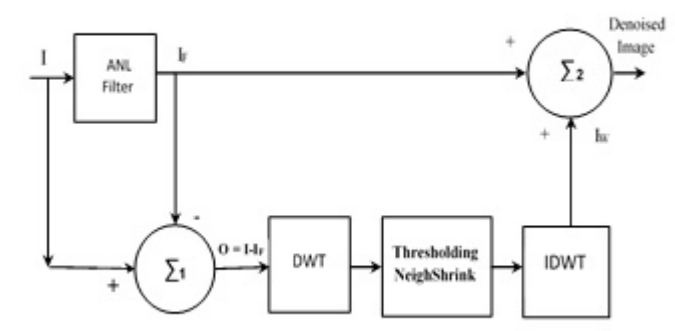

Fig 2. Block diagram of ANL with Method Noise Thresholding

In this process, the noisy image (I) is applied to ANL filter to obtain a pre-filtered image $\left(\mathrm{I}_{F}\right)$. Method noise $(\mathrm{O})$ is obtained by computing the difference of pre-filtered image $\left(\mathrm{I}_{F}\right)$ and noisy image (I) through summing block-1. Method noise applied to discrete wavelet transform (DWT) block to obtain three level decomposed images of method noise. NeighShrinksure thresholding eliminates the noisy components of the DWT output. Inverse discrete wavelet transform (IDWT) ( $I_{W}$ ) recovers the image features present in the method noise. The $\mathrm{I}_{F}$ image and image features $\mathrm{I}_{W}$ are combined in summing block-2 to obtain the denoised image. Figure 2 shows the block diagram representation.

\section{Results and Discussions}

The newly developed denoising algorithm implemented through MATLAB software compares with other denoising algorithms. A standard 256 x 256 grayscale images denoised using Wavelet Thresholding (WT), Gaussian Bilateral Filter with Method Noise Thresholding (GBFMT) and Weiner Filter with Residual noise Thresholding (WFRT) methods is compared with proposed 
denoising algorithm. Standard images of Lena, Barbara, and Girl face considered in the proposed work are shown in Figure 3. These standard images obtained from the image database (http://www.imageprocessingplace.com/root_files_V3/image_data bases.htm). The noisy image obtained by adding additive Gaussian noise to the standard images with standard deviation 10, 20, 30, 40, and 50. The noisy images denoised using WT, GBFMT, WFRT and proposed method ANLMNT algorithm are used to obtain PSNR and SSIN parameters. DWT block provides three levels of decomposition of the noisy images using Bior6.8 wavelet. The denoised image of the proposed filter compared with GBFMT and WFRT methods show improvement in PSNR and SSIN parameters. Image Quality Indices (IQI) parameter of different noisy images is obtained. (Figure 3)

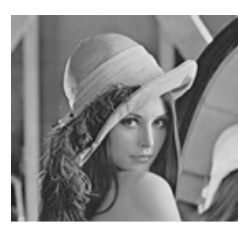

(a)

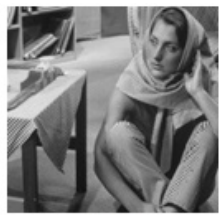

(b)

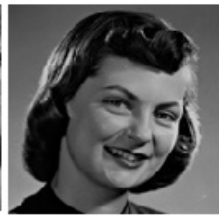

(c)

Fig 3. Standard Images a) Leng.png b) Barbara.png c) Girlface.png

\subsection{Performance Matrices}

\subsubsection{Peak Signal to Noise Ratio (PSNR)}

Refers to the ratio of maximum possible power of a signal to the power of corrupting noise.

$$
P S N R=10 \log \left(\frac{255}{M S E}\right)^{2}
$$

where, MSE refers to Mean Sample Error of the pixel throughout the image.

\subsubsection{Structural Similarity Index (SSIN)}

The similarity between the original image and the image obtained by the denoising technique is the structural similarity index. It is a perception-based model of an image, and change of perception in structural information of an image is referred as image degradation. Image degradation may be due to luminance and contrast masking. Distortions measured at the edges of the denoised image are the luminance masking, and distortions in an image's texture are contrast masking. The structural similarity index is given by (22).

$$
\operatorname{SSIM}(x, y)=\frac{\left(2 \mu_{x} \mu_{y}+C_{1}\right)\left(2 \sigma_{x y}+C_{2}\right)}{\left(\mu_{x}^{2}+\mu_{y}^{2}+C_{1}\right)\left(\sigma_{x}^{2}+\sigma_{y}^{2}+C_{2}\right)}
$$

Where $\mu_{\mathrm{x}}$ and $\mu_{\mathrm{y}}$ denotes the mean values of original and distorted images, $\sigma_{\mathrm{x}}$ and $\sigma_{\mathrm{y}}$ represents the standard deviation of the original and distorted images, is the covariance of both images, $\mathrm{C}_{1}$ and $\mathrm{C}_{2}$ are constants.

\subsubsection{Image Quality Index (IQI)}

This parameter (IQI) is obtained by modeling the image distortion related to the reference image with loss correlation, luminance distortion, and contrast distortion.

$$
\mathrm{IQI}(x, y)=\frac{\left(4 \mu_{x} \mu_{y} \mu_{x y}\right)}{\left(\mu_{x}^{2}+\mu_{y}^{2}\right)\left(\sigma_{x}^{2}+\sigma_{y}^{2}\right)}
$$

\subsection{Experimental Results}

PSNR and SSIN parameters of the denoised image using ANLMNT (proposed method) are compared with WT, GBFMT and WFRT methods. Table 1 shows the tabulation of PSNR values of ANLMNT and other thresholding techniques. The method noise thresholding technique incorporated along with ANL filter in the present study show better PSNR as compared to GBFMT and WFRT methods. 
Table 1. Comparison of PSNR values of ANLMNT with GBFMT and WFRT

\begin{tabular}{|c|c|c|c|c|c|}
\hline$\sigma$ & 10 & 20 & 30 & 40 & 50 \\
\hline Method & \multicolumn{5}{|c|}{ Input Image(LENA) } \\
\hline WT & 27.36 & 24.97 & 23.97 & 23.41 & 22.90 \\
\hline GBFMT & 33.07 & 29.23 & 27.19 & 25.76 & 24.69 \\
\hline WFRT & 33.23 & 28.94 & 26.71 & 25.13 & 23.79 \\
\hline \multirow[t]{2}{*}{ ANLMNT (Proposed Method) } & 33.80 & 30.34 & 28.11 & 26.32 & 24.75 \\
\hline & \multicolumn{5}{|c|}{ Input Image(BARBARA) } \\
\hline WT & 26.33 & 24.27 & 23.28 & 22.69 & 22.29 \\
\hline GBFMT & 31.78 & 28.33 & 26.53 & 25.27 & 24.26 \\
\hline WFRT & 32.10 & 28.31 & 26.21 & 24.72 & 23.48 \\
\hline \multirow[t]{2}{*}{ ANLMNT (Proposed Method) } & 32.54 & 29.18 & 27.18 & 25.59 & 24.13 \\
\hline & \multicolumn{5}{|c|}{ Input Image(GIRLFACE) } \\
\hline WT & 30.72 & 26.03 & 24.80 & 23.92 & 23.07 \\
\hline GBFMT & 33.71 & 29.59 & 27.09 & 25.22 & 23.84 \\
\hline WFRT & 32.78 & 28.14 & 25.66 & 23.85 & 22.43 \\
\hline ANLMNT (Proposed Method) & 34.62 & 30.62 & 27.99 & 25.89 & 24.20 \\
\hline
\end{tabular}

The proposed ANLMNT denoising method depicts an improvement in PSNR compared to other denoising techniques. However PSNR value decreases as sigma increases $(\sigma>30)$. A study with high contrast standard image (Girlface) shows that the proposed work has significantly improved PSNR values compared to other standard images with low contrast (Lena and Barbara). The proposed method also show increase in SSIN values of the standard images (Table 2).

Table 2. Comparison of SSIN values for proposed method with GBFMT and WFRT

\begin{tabular}{llllll}
\hline$\sigma$ & 10 & 20 & 30 & 40 & 50 \\
\hline Method & Input Image(LENA) & & & & \\
\hline WT & 0.9989 & 0.9985 & 0.9983 & 0.9982 & 0.9981 \\
GBFMT & 0.9996 & 0.9992 & 0.9989 & 0.9986 & 0.9984 \\
WFRT & 0.9996 & 0.9992 & 0.9989 & 0.9986 & 0.9982 \\
ANLMNT (Proposed Method) & 0.9996 & 0.9994 & 0.9991 & 0.9988 & 0.9985 \\
\hline
\end{tabular}

Table 3 shows the IQI values of the ANLMNT method for standard images.

Table 3. Comparison of IQI values for different Images

\begin{tabular}{llllll}
\hline$\sigma$ & 10 & 20 & 30 & 40 & 50 \\
\hline LENA & 0.9950 & 0.9893 & 0.9816 & 0.9734 & 0.9647 \\
BARBARA & 0.9930 & 0.9853 & 0.9784 & 0.9686 & 0.9585 \\
GIRL FACE & 0.9956 & 0.9875 & 0.9774 & 0.9668 & 0.9572 \\
\hline
\end{tabular}

Figure 4(a) shows the Lena image corrupted with standard noise $\sigma=20$. From Figure 4(b, $c, d)$, it can be seen that WT blurred the image, GBFMT introduces artifacts, WFRT introduces some white patches in the denoised image. Figure 4e shows the image denoised with the proposed method having better quality as compared to other above-compared methods while preserving image features. The proposed method is also applied to denoise other standard images with $\sigma=10,20,30,40$, and 50, like Barbara and Girlface are shown in Figures 5 and 6. Table 4 shows the comparison of different noise filter methods with $=10$ relating PSNR and SSIN image parameters. 
Table 4. Comparison of PSNR values for different Methods

\begin{tabular}{lllll}
\hline Noise Filters with $\sigma=20$ Image Parameters & GBFMT [5] & WFRT[6] & FAN [7] & ANLMNT (Proposed Work) \\
\hline PSNR & 29.23 & 28.94 & 30.46 & 30.34 \\
SSIN & 0.9935 & 0.9992 & 0.851 & 0.9994 \\
\hline
\end{tabular}

\subsection{Discussion:}

Method Noise Thresholding (MNT) technique implemented through Gaussian Bilateral filter and Wiener Filter with Residual Thresholding shows lesser PSNR and SSIN values when implemented through Adaptive Non Local mean filter. Table 4 shows, GBFMT and WFRT filtering methods with lesser PSNR values that is 33.07 and 33.23 as compared to PSNR value of 33.80 in the proposed work. Increase in SSIN is also observed in this method. GBFMT ${ }^{(5)}$ filter results in artifacts and WFRT ${ }^{(6)}$ filter provides less PSNR value at higher ' $\sigma$ '. These drawbacks are overcome in the proposed work. These results are analyzed with noise standard $(\sigma=10)$ using bior6.8 wavelet.

Comparison Table 4 shows that there is drastic improvement in PSNR value 0.7 when compared with Priya et al. ${ }^{(6)}$ and slight improvement in SSIM 0.003. In case of Fan et al. and Hernández-Gutiérrez ${ }^{(8,9)}$ even though PSNR remains almost same but there is drastic improvement in SSIN value 0.148. Hence, the proposed method shows improvement in both PSNR and SSIN values as compared with the results obtained with similar methods in recent years.

The proposed denoising technique studied for standard Lena image with varying sigma values and wavelets shows improvement in PSNR value for lower sigma values $(10,20)$ and for higher values of sigma, it is constant. This reveals that proposed ANLMNT technique is more suitable for images with lower sigma values. In addition, PSNR increases with bior6.8 wavelet as compared to other wavelets (Table 5).

Table 5. Comparison of PSNR values for different Wavelets

\begin{tabular}{llllll}
\hline Wavelet & Db8 & Sym8 & Db16 & Coif5 & Bior6.8 \\
\hline$\sigma$ & \multicolumn{6}{l}{ PSNR Value } & & & \\
\hline 10 & 33.75 & 33.77 & 33.75 & 33.77 & 33.80 \\
20 & 30.31 & 30.32 & 30.29 & 30.32 & 30.34 \\
30 & 28.10 & 28.11 & 28.06 & 28.12 & 28.11 \\
40 & 26.32 & 26.33 & 26.30 & 26.31 & 26.32 \\
50 & 24.77 & 24.77 & 24.74 & 24.75 & 24.75 \\
\hline
\end{tabular}

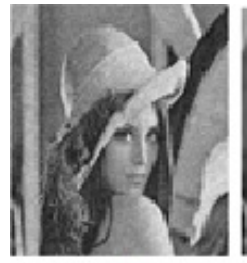

(a)

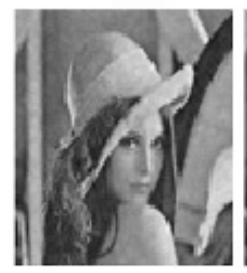

(d)

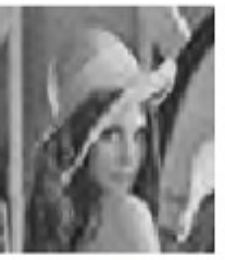

(b)

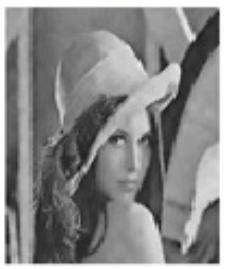

(e)

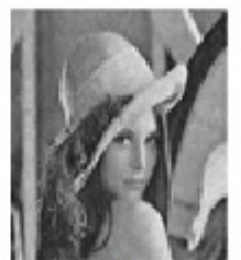

(c)

Fig 4. (a) Noisy Image $\sigma=20$ b) WT c) GBFMT d) WFRT e) ANLMNT 


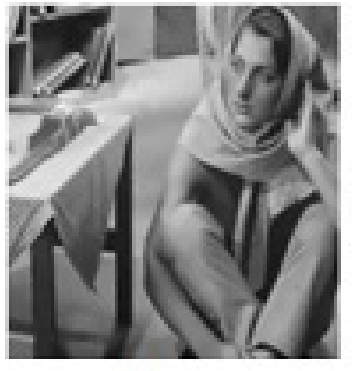

(a)

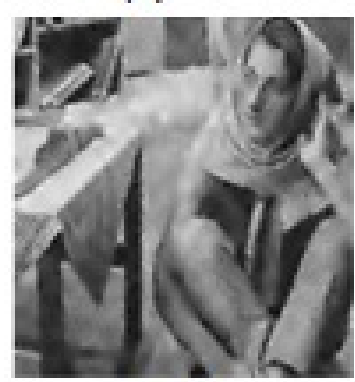

(d)

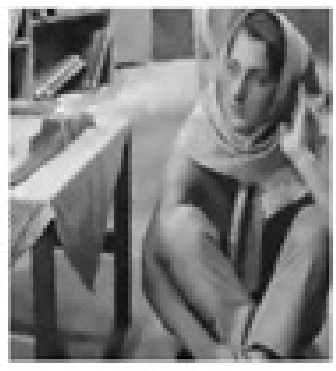

(b)

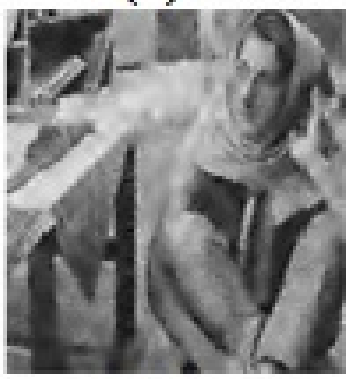

(e)

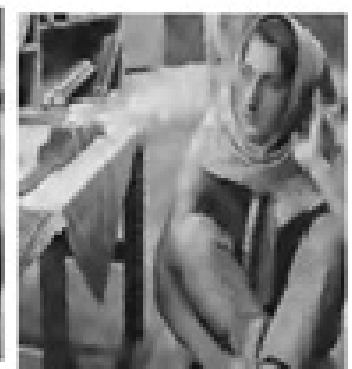

(c)

Fig 5. (a to e) Barbara image Denoised with proposed method for $\sigma=10,20,30,40$ and 50

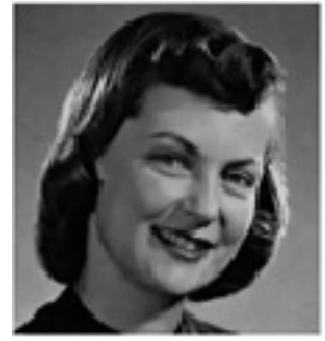

(a)

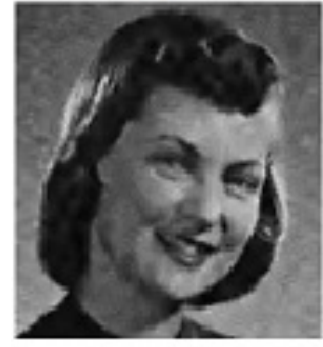

(d)

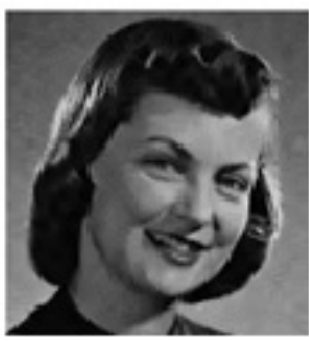

(b)

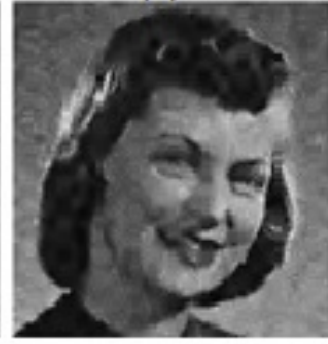

(e)

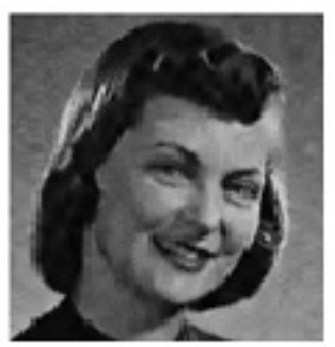

(c)

Fig 6. (a to e) Girlface image Denoised with proposed method for $\sigma=10,20,30,40$ and 50 


\section{Conclusion}

An Adaptive NL means of filtering with method noise thresholding (ANLMNT) technique is proposed in this work. Simulation results of the present method depict that the denoised images have improved PSNR and SSIN values when compared with other method noise thresholding techniques. The proposed work is best suited for images with high noise and high contrast. Finetuning at the pre-filtering stage may further enhance the quality of the image. Implementation of other denoising techniques using method of noise thresholding may result in improved denoised image. PSNR value substantially reduces for higher noise levels $(\sigma>30)$. There is scope for refinement of image parameters through improved filtering methods for higher $\sigma$ values. Further, the obtained denoised images through novel filtering technique have fewer artifacts and efficient at higher noise levels.

\section{References}

1) Rana A, Pathak C, and. Wavelet Thresholding Algorithms for Image Denoising. Indian Journal of Science and Technology. 2018;11(27):1-6. Available from: https://dx.doi.org/10.17485/ijst/2018/v11i27/130706.

2) Gouho JBB. Automatic Modulation Classification Based on In-Phase Quadrature Diagram Constellation Combined with a Deep Learning Model. Indian Journal of Science and Technology. 2020;13(2):200-212. Available from: https://dx.doi.org/10.17485/ijst/2020/v13i02/148648.

3) Chen K, Lin X, Hu X, Wang J, Zhong H, Jiang L. An enhanced adaptive non-local means algorithm for Rician noise reduction in magnetic resonance brain images. BMC Medical Imaging. 2020;20(1). Available from: https://dx.doi.org/10.1186/s12880-019-0407-4.

4) Kang SH, Kim JY. Application of Fast Non-Local Means Algorithm for Noise Reduction Using Separable Color Channels in Light Microscopy Images. International Journal of Environmental Research and Public Health. 2021;18(6):1-12. Available from: https://dx.doi.org/10.3390/ijerph18062903.

5) Kumar B. Image denoising based on gaussian/bilateral filter and its method noise thresholding. Signal, Image Video Processing. $2013 ; 7(6): 1159-1172$. Available from: http://dx.doi.org/10.1007/s11760-012-0372-7.

6) Priya BS, Jagadale BN, Naragund MN, Hegde V, Panchaxri. An Efficient Image Denoising Based on Weiner Filter and Neigh Sure Shrink. International Journal of Innovative Technology and Exploring Engineering. 2019;9(2):76-80. doi:10.35940/ijitee.a4905.129219.

7) Verma R, Pandey R. Non local means algorithm with adaptive isotropic search window size for image denoising. 2015 Annual IEEE India Conference (INDICON). 2015;4(3):1-5. doi:10.1109/INDICON.2015.7443193.

8) Linwei F, Xuemei L, Qiang G, Caiming Z. Nonlocal image denoising using edge-based similarity metric and adaptive parameter selection. Science China Information Sciences. 2018;p. 61. Available from: https://doi.org/10.1007/s11432-017-9207-9.

9) Hernández-Gutiérrez IV, Gallegos-Funes FJ, Rosales-Silva AJ. Improved preclassification non local-means (IPNLM) for filtering of grayscale images degraded with additive white Gaussian noise. EURASIP Journal on Image and Video Processing. 2018;104(1). Available from: https://dx.doi.org/10.1186/ s13640-018-0346-y. 